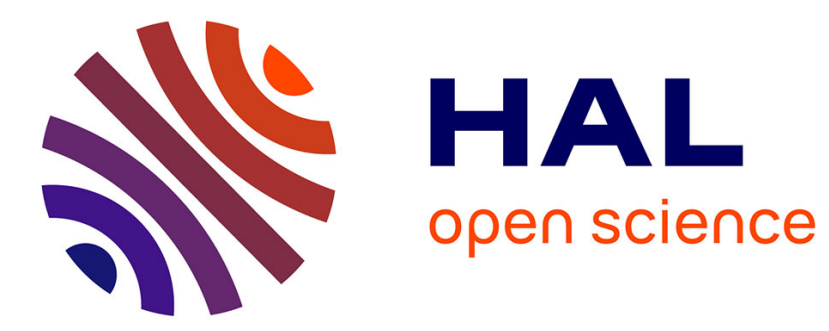

\title{
Howe's Method for Calculi with Passivation
}

Sergueï Lenglet, Alan Schmitt, Jean-Bernard Stefani

\section{To cite this version:}

Sergueï Lenglet, Alan Schmitt, Jean-Bernard Stefani. Howe's Method for Calculi with Passivation. 20th International Conference on Concurrency Theory (CONCUR 2009), Sep 2009, Bologna, Italy. pp.448-462. inria-00486800

\section{HAL Id: inria-00486800 https://hal.inria.fr/inria-00486800}

Submitted on 26 May 2010

HAL is a multi-disciplinary open access archive for the deposit and dissemination of scientific research documents, whether they are published or not. The documents may come from teaching and research institutions in France or abroad, or from public or private research centers.
L'archive ouverte pluridisciplinaire HAL, est destinée au dépôt et à la diffusion de documents scientifiques de niveau recherche, publiés ou non, émanant des établissements d'enseignement et de recherche français ou étrangers, des laboratoires publics ou privés. 


\title{
Howe's Method for Calculi with Passivation
}

\author{
Sergueï Lenglet ${ }^{1}$, Alan Schmitt ${ }^{2}$, and Jean-Bernard Stefani ${ }^{2}$ \\ 1 Université Joseph Fourier, Grenoble, France \\ 2 INRIA Rhône-Alpes, France
}

\begin{abstract}
We show how to apply Howe's method for the proof of congruence of early bisimilarities in a higher-order process calculus with passivation. This involves the introduction of a proof technique based on a new kind of transition system and bisimilarity, called complementary semantics. We show that complementary semantics is equivalent to contextual semantics, originally introduced by Sangiorgi, that relies on classical transition systems for higher-order calculi and context bisimilarity. We apply this technique to obtain the first characterization of weak barbed congruence for such a higher-order calculus with passivation.
\end{abstract}

\section{Introduction}

Motivation. A natural notion of program equivalence in concurrent languages is a form of contextual equivalence called barbed congruence, introduced by Milner and Sangiorgi [17]. Roughly, given an operational semantics defined by means of a small-step reduction relation, two processes are barbed congruent if they have the same reductions and the same observables (or barbs), under any context.

The definition of barbed congruence, however, is impractical to use in proofs because of its quantification on contexts. An important question, therefore, is to find more effective characterizations of barbed congruence. A powerful method for proving program equivalence is the use of coinduction with the definition of an appropriate notion of bisimulation. The question of characterizing barbed congruence to enable the use of coinduction becomes that of finding appropriate bisimulation relations such that their resulting behavioral equivalences, called bisimilarities, are sound (i.e., included in barbed congruence) and complete (i.e., containing barbed congruence) with respect to barbed congruence.

For first-order languages, such as CCS or the $\pi$-calculus, the behavioral theory and the associated proof techniques, e.g., for proving congruence, are well developed [23]. Characterizing barbed congruence in these languages is a reasonably well understood proposition. The situation is less satisfactory for higherorder concurrent languages. Bisimilarity relations that coincide with barbed congruence have only been given for some higher-order concurrent languages. They usually take the form of context bisimilarities, building on a notion of context bisimulation introduced by Sangiorgi for a higher-order $\pi$-calculus, $\mathrm{HO} \pi$ [21]. Context bisimilarity has been proven to coincide with barbed congruence for higher-order variants of the $\pi$-calculus: Sangiorgi's $\mathrm{HO} \pi[20,21,12]$, a concurrent ML with local names [11], a higher-order distributed $\pi$-calculus called 
SafeDpi [8], Mobile Ambients [16], and some of Mobile Ambients's variants such as Boxed Ambients [3]. A sound but incomplete form of context bisimilarity has been proposed for the Seal calculus [5]. For the Homer calculus [6], strong context bisimilarity is proven sound and complete, but weak context bisimilarity is not complete. A sound and complete context bisimilarity has been defined for the Kell calculus [24], but for the strong case only.

The key step in proving the soundness of candidate bisimilarities in higherorder concurrent calculi is to show that they are congruences. A systematic technique for proving the congruence of bisimilarity relations is Howe's method $[10,1,7]$. Unfortunately, Howe's method is originally well suited for bisimulations that are defined in both a late and a delay style, either of which generally breaks the correspondence with barbed congruence. For Homer, Howe's method has been extended to a version of context bisimulation in an input-early style [6], but the resulting weak bisimilarity is not complete with respect to weak barbed congruence.

Contributions. In this paper, we show how to apply Howe's method to deal with bisimulations defined in an early (and non-delay) style. This involves the introduction of complementary semantics, a labelled transition system and its associated bisimulation. This semantics is designed to avoid the key difficulty in applying Howe's method to a bisimulation defined in an early style. We use complementary semantics as a proof technique to obtain a characterization of weak barbed congruence in a concurrent calculus called $\mathrm{HO} \pi \mathrm{P}$. $\mathrm{HO} \pi \mathrm{P}$ is a calculus introduced in [15] to study the behavioral theory of passivation in a simpler setting than in Homer [9] or the Kell calculus [24]. Passivation allows a named locality to be stopped and its state captured for further handling. It can be used to model process failures, strong process mobility, and "thread thunkification" as in the Acute programming language [25] (see [24] for discussion and motivation). To our knowledge, this is the first characterization of weak barbed congruence for a concurrent calculus with both restriction and passivation.

Outline. In Section 2, we present the syntax, contextual semantics, and known bisimilarity results for $\mathrm{HO} \pi \mathrm{P}$. In Section 3, we explain why Howe's method fails with early context bisimilarities, and present the intuition behind our approach. We propose in Section 4 a new semantics and associated bisimilarities for $\mathrm{HO} \pi \mathrm{P}$, called complementary semantics. We prove that the semantics are equivalent, that complementary bisimilarity coincides with early context bisimilarity, and that complementary bisimilarity is a congruence using Howe's method. We discuss related work in Section 5. Section 6 concludes the paper. Proofs and additional details are available in the draft of the full paper [14].

\section{$2 \mathrm{HO} \pi \mathrm{P}$ Contextual Semantics}

$\mathrm{HO} \pi \mathrm{P}$ (Higher-Order $\pi$ with Passivation) [15] extends the higher-order calculus $\mathrm{HO} \pi[21]$ with localities $a[P]$, that are passivation units. We write names $a, b \ldots$, 


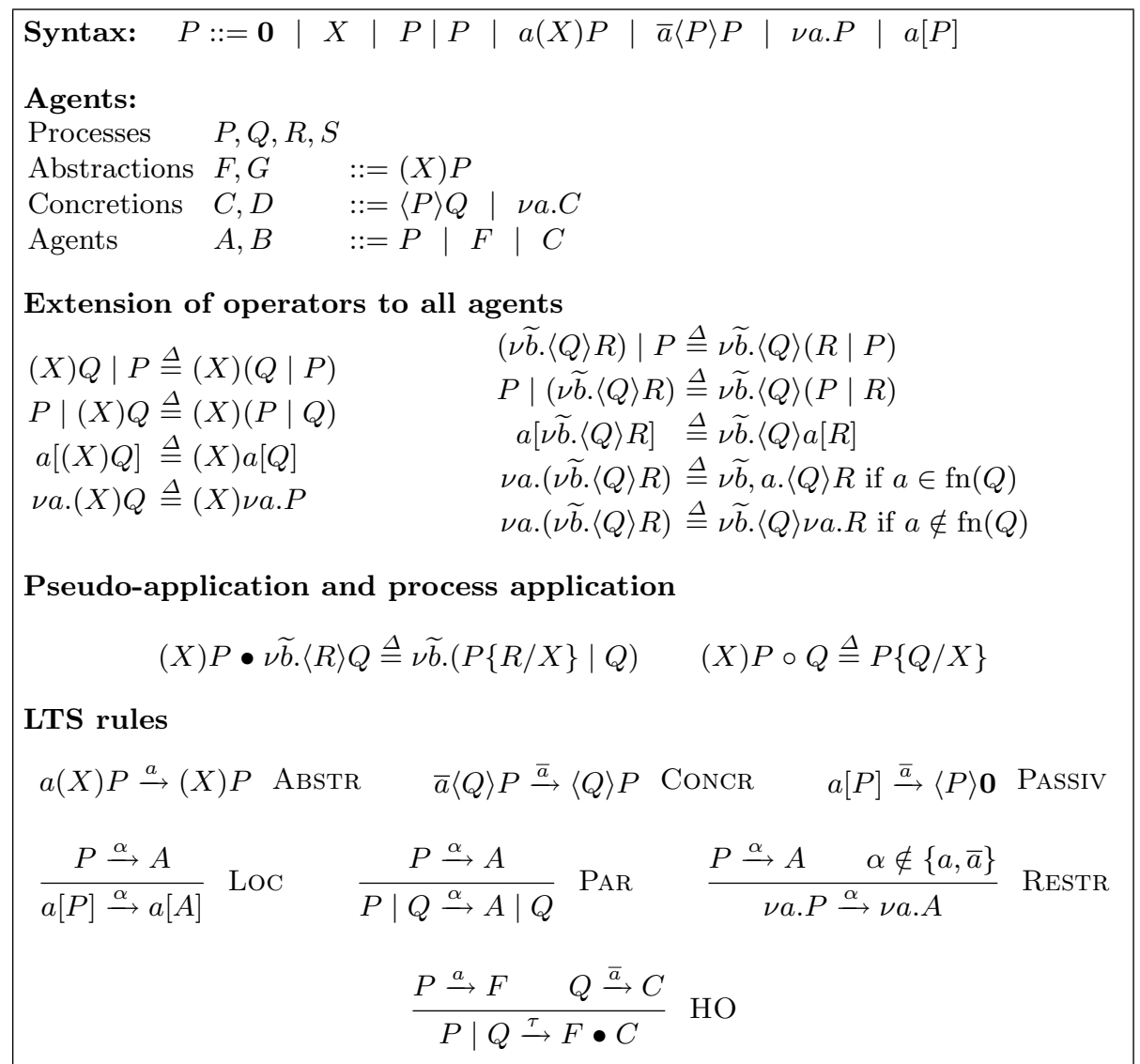

Fig. 1. Contextual Labeled Transition System for $\mathrm{HO} \pi \mathrm{P}$

conames $\bar{a}, \bar{b} \ldots$, and process variables $X, Y, \ldots$ Sets $\left\{x_{1} \ldots x_{n}\right\}$ are written $\widetilde{x}$. We let $\gamma$ range over names and conames.

Convention. We identify processes up to $\alpha$-conversion of names and variables: processes and agents are always chosen such that their bound names and variables are distinct from free names and variables. We also assume that bound names and variables of any process or actions under consideration are chosen to be different from free names and variables of any other entities under consideration. Note that with this convention, we have $(X) Q \mid P \triangleq(X)(Q \mid P)$ without qualification on the free variables of $P$.

Syntax and contextual semantics of the calculus can be found in Figure 1, with the exception of the symmetric rules for PAR and HO. In process $a(X) P$, the variable $X$ is bound. Similarly, in process $\nu a . P$, the name $a$ is bound. We write $\mathrm{fv}(P)$ for the free variables of a process $P, \mathrm{fn}(P)$ for its free names, and $\operatorname{bn}(P)$ for its bound names. We write $P\{Q / X\}$ for the capture-free substitution of $X$ by $Q$ in $P$. A closed process has no free variable. 
Processes may evolve towards a process (internal actions $P \stackrel{\tau}{\rightarrow} P^{\prime}$ ), an $a b$ straction (message input $P \stackrel{a}{\rightarrow} F=(X) Q$ ), or a concretion (message output $P \stackrel{\bar{a}}{\rightarrow} C=\nu \widetilde{b} .\langle R\rangle Q)$. Transition $P \stackrel{a}{\rightarrow}(X) Q$ means that $P$ may receive process $R$ on $a$ to continue as $Q\{R / X\}$. Transition $P \stackrel{\bar{a}}{\rightarrow} \nu \widetilde{b} \cdot\langle R\rangle Q$ means that $P$ may send process $R$ on $a$ and continue as $Q$, and the scope of names $\widetilde{b}$ has to be expanded to encompass the recipient of $R$. A synchronous higher-order communication takes place when a concretion interacts with an abstraction (rule HO).

A locality $a[P]$ is a transparent evaluation context: process $P$ may evolve by itself and communicate freely with processes outside of locality $a$ (rule LOC). At any time, passivation may be triggered and locality $a[P]$ becomes a concretion $\langle P\rangle \mathbf{0}$ (rule PASsiv). Rule Loc implies that the scope of restricted names may cross locality boundaries. Scope extrusion outside localities is performed "by need" when a communication takes place, as defined in the extension of restriction to concretions in Fig. 1. Note that with this semantics, the interaction between passivation and restriction is not benign: in general processes $b[\nu a . P]$ and $\nu a . b[P]$ are not barbed congruent (see [14] for more details).

Remark 1. In $\mathrm{HO} \pi \mathrm{P}$, process $a(X) P$ is used for message input and process passivation, while Homer and the Kell calculus use two different receiving patterns. We chose to keep $\mathrm{HO} \pi \mathrm{P}$ syntax as simple as possible; adding a specific input for passivation does not change our results.

\section{Contextual Equivalences}

Barbed congruence is the usual reduction-based behavioral equivalence. We identify reduction with $\tau$-transition $\longrightarrow \stackrel{\Delta}{\rightarrow}$ and define weak reduction $\Longrightarrow$ as the reflexive and transitive closure of $\longrightarrow$. Observables $\gamma$ of a process $P$, written $P \downarrow_{\gamma}$, are unrestricted names or conames on which a communication may immediately occur $(P \stackrel{\gamma}{\rightarrow} A$, for some agent $A)$. Contexts $\mathbb{C}$ are terms with a hole $\square$. A relation $\mathcal{R}$ is a congruence iff $P \mathcal{R} Q$ implies $\mathbb{C}\{P\} \mathcal{R} \mathbb{C}\{Q\}$ for all contexts $\mathbb{C}$.

Definition 1. A symmetric relation $\mathcal{R}$ on closed processes is a strong (resp. weak) barbed bisimulation iff $P \mathcal{R} Q$ implies:

$$
\begin{aligned}
& \text { - for all } P \downarrow_{\gamma} \text {, we have } Q \downarrow_{\gamma}\left(\text { resp. } Q \Longrightarrow \downarrow_{\gamma}\right) \text {; } \\
& \text { - for all } P \longrightarrow P^{\prime} \text {, there exists } Q^{\prime} \text { such that } Q \longrightarrow Q^{\prime}\left(\text { resp. } Q \Longrightarrow Q^{\prime}\right) \text { and } \\
& \quad P^{\prime} \mathcal{R} Q^{\prime} ;
\end{aligned}
$$

Strong (resp. weak) barbed congruence $\sim_{b}\left(\right.$ resp. $\left.\approx_{b}\right)$ is the largest congruence that is a strong (resp. weak) barbed bisimulation.

A relation $\mathcal{R}$ is sound with respect to $\sim_{b}\left(\right.$ resp. $\left.\approx_{b}\right)$ iff $\mathcal{R} \subseteq \sim_{b}\left(\right.$ resp. $\left.\mathcal{R} \subseteq \approx_{b}\right) ; \mathcal{R}$ is complete with respect to $\sim_{b}$ (resp. $\approx_{b}$ ) iff $\sim_{b} \subseteq \mathcal{R}$ (resp. $\approx_{b} \subseteq \mathcal{R}$ ).

As in $\mathrm{HO} \pi[21]$, we characterize strong barbed congruence using an early strong context bisimilarity. As explained in [15], bisimilarities in $\mathrm{HO} \pi \mathrm{P}$ require 
more discriminating power than in $\mathrm{HO} \pi$, as the passivation of enclosing localities has to be taken into account. Let bisimulation contexts $\mathbb{E}$ be evaluation contexts, i.e., contexts that allow transitions at the hole position, used for observational purposes.

$$
\mathbb{E}::=\square|\nu a . \mathbb{E}| \mathbb{E}|P| P|\mathbb{E}| a[\mathbb{E}]
$$

We write bn $(\mathbb{E})$ the names bound at the hole position by the context $\mathbb{E}$ : a name $a \in \operatorname{bn}(\mathbb{E}) \cap \mathrm{fn}(P)$ is free in $P$ and becomes bound in $\mathbb{E}\{P\}$.

Definition 2. Early strong context bisimilarity $\sim$ is the largest symmetric relation on closed processes $\mathcal{R}$ such that $P \mathcal{R} Q$ implies $f n(P)=f n(Q)$ and:

- for all $P \stackrel{\tau}{\rightarrow} P^{\prime}$, there exists $Q^{\prime}$ such that $Q \stackrel{\tau}{\rightarrow} Q^{\prime}$ and $P^{\prime} \mathcal{R} Q^{\prime}$;

- for all $P \stackrel{a}{\rightarrow} F$, for all $C$, there exists $F^{\prime}$ such that $Q \stackrel{a}{\rightarrow} F^{\prime}$ and $F \bullet C \mathcal{R}$ $F^{\prime} \bullet C$;

- for all $P \stackrel{\bar{a}}{\rightarrow} C$, for all $F$, there exists $C^{\prime}$ such that $Q \stackrel{\bar{a}}{\rightarrow} C^{\prime}$ and for all $\mathbb{E}$, $F \bullet \mathbb{E}\{C\} \mathcal{R} F \bullet \mathbb{E}\left\{C^{\prime}\right\}$.

Note. The late variant of strong context bisimulation can simply be obtained by changing the order of quantifications on concretions and abstractions in the above clauses. Thus the clause for input in late style would be: for all $P \stackrel{a}{\rightarrow} F$, there exists $F^{\prime}$ such that $Q \stackrel{a}{\rightarrow} F^{\prime}$ and for all $C$, we have $F \bullet C \mathcal{R} F^{\prime} \bullet C$.

Condition $\mathrm{fn}(P)=\mathrm{fn}(Q)$ is required because of lazy scope extrusion: two bisimilar processes with different free names may be distinguished by scope extrusion and passivation $[15,13]$.

Theorem 1. Relation $\sim$ is a congruence and $\sim=\sim_{b}$.

The proof of congruence in Theorem 1 is the same as in the Kell calculus [24] (see [14] for details). Unfortunately, this technique fails with weak relations. We write $\stackrel{\tau}{\Rightarrow}$ for the reflexive and transitive closure of $\stackrel{\tau}{\rightarrow}$. For every higher-order name or coname $\gamma$, we write $\stackrel{\gamma}{\Rightarrow}$ for $\Rightarrow \stackrel{\gamma}{\rightarrow}$. As higher order steps result in concretions and abstractions, they may not reduce further; silent steps after this reduction are taken into account in the definition of weak bisimulation.

Definition 3. Early weak context bisimilarity $\approx$ is the largest symmetric relation on closed processes $\mathcal{R}$ such that $P \mathcal{R} Q$ implies $f n(P)=f n(Q)$ and:

- for all $P \stackrel{\tau}{\rightarrow} P^{\prime}$, there exists $Q^{\prime}$ such that $Q \stackrel{\tau}{\Rightarrow} Q^{\prime}$ and $P^{\prime} \mathcal{R} Q^{\prime}$;

- for all $P \stackrel{a}{\rightarrow} F$, for all $C$, there exist $F^{\prime}, Q^{\prime}$ such that $Q \stackrel{a}{\Rightarrow} F^{\prime}, F^{\prime} \bullet C \stackrel{\tau}{\Rightarrow} Q^{\prime}$, and $F \bullet C \mathcal{R} Q^{\prime}$;

- for all $P \stackrel{\bar{a}}{\rightarrow} C$, for all $F$, there exists $C^{\prime}$ such that $Q \stackrel{\bar{a}}{\Rightarrow} C^{\prime}$ and for all $\mathbb{E}$, there exists $Q^{\prime}$ such that $F \bullet \mathbb{E}\left\{C^{\prime}\right\} \stackrel{\tau}{\Rightarrow} Q^{\prime}$ and $F \bullet \mathbb{E}\{C\} \mathcal{R} Q^{\prime}$.

Barbed congruence and context bisimilarities are extended to open processes via the notion of open extension $\mathcal{R}^{\circ}$ of a relation $\mathcal{R}$ on closed processes.

Definition 4. Let $P$ and $Q$ be two open processes. We have $P \mathcal{R}^{\circ} Q$ iff $P \sigma \mathcal{R}$ $Q \sigma$ for all process substitutions $\sigma$ that close $P$ and $Q$.

In the following section, we present a congruence proof technique, called Howe's method, and then explain why it fails with early context bisimilarities. 


\section{Howe's Method}

The essence of the method. Howe's method $[10,1,7]$ is a systematic proof technique to show that a bisimilarity $\mathcal{R}$ (and its open extension $\mathcal{R}^{\circ}$ ) is a congruence. The method can be divided in three steps: first, prove some basic properties on the Howe's closure $\mathcal{R}^{\bullet}$ of the relation. By construction, $\mathcal{R}^{\bullet}$ contains $\mathcal{R}^{\circ}$ and is a congruence. Second, prove a simulation-like property for $\mathcal{R}^{\bullet}$. Finally, prove that $\mathcal{R}$ and $\mathcal{R}^{\bullet}$ coincide on closed processes. Since $\mathcal{R}^{\bullet}$ is a congruence, conclude that $\mathcal{R}$ is a congruence.

Howe's closure is inductively defined as the smallest congruence which contains $\mathcal{R}^{\circ}$ and is closed under right composition with $\mathcal{R}^{\circ}$.

Definition 5. Howe's closure $\mathcal{R}^{\bullet}$ of a relation $\mathcal{R}$ is the smallest relation verifying:

$-\mathcal{R}^{\circ} \subseteq \mathcal{R}^{\bullet}$

$-\mathcal{R}^{\bullet} \overline{\mathcal{R}}^{\circ} \subseteq \mathcal{R}^{\bullet}$

- for all operators op of the language, if $\widetilde{P} \mathcal{R} \bullet \widetilde{Q}$, then op $(\widetilde{P}) \mathcal{R} \bullet$ op $(\widetilde{Q})$.

By definition, $\mathcal{R}^{\bullet}$ is a congruence, and the composition with $\mathcal{R}^{\circ}$ allows some transitivity and gives additional properties to the relation.

Remark 2. In the literature (e.g., $[10,7,9]$ ) Howe's closure is usually inductively defined by the following rule for all operators op in the language:

$$
\frac{\widetilde{P} \mathcal{R}^{\bullet} \widetilde{R} \quad o p(\widetilde{R}) \mathcal{R}^{\circ} Q}{o p(\widetilde{P}) \mathcal{R}^{\bullet} Q}
$$

Both definitions are equivalent (see [7] for the proof). We believe that Definition 5 is easier to understand and to work with in the proofs.

By definition, we have $\mathcal{R}^{\circ} \subseteq \mathcal{R}^{\bullet}$. To have the reverse inclusion, we prove that $\mathcal{R}^{\bullet}$ is a bisimulation. To this end, we need the following classical properties of Howe's closure of any equivalence $\mathcal{R}$.

Lemma 1. Let $\mathcal{R}$ be an equivalence.

- If $P \mathcal{R}^{\bullet} Q$ and $R \mathcal{R}^{\bullet} S$, then we have $P\{R / X\} \mathcal{R}^{\bullet} Q\{S / X\}$.

- The reflexive and transitive closure $\left(\mathcal{R}^{\bullet}\right)^{*}$ of $\mathcal{R}^{\bullet}$ is symmetric.

The first property is typically used to establish the simulation-like result (second step of the method). Then one proves that the restriction of $\left(\mathcal{R}^{\bullet}\right)^{*}$ to closed terms is a bisimulation. Consequently we have $\mathcal{R} \subseteq \mathcal{R} \bullet \subseteq\left(\mathcal{R}^{\bullet}\right)^{*} \subseteq \mathcal{R}$ on closed terms, and we conclude that bisimilarity $\mathcal{R}$ is a congruence.

The main difficulty of the method lies in the proof of the simulation-like property for Howe's closure. In the following, we explain why we cannot directly use Howe's method with early context bisimilarity (Definition 2). 
Communication problem. To prove that $\mathcal{R}^{\bullet}$ is a simulation, we need to establish a stronger result, to avoid transitivity issues which otherwise would arise and make the method fail in the weak case [14]. Given a bisimilarity $\mathcal{R}$ based on a LTS $P \stackrel{\lambda}{\rightarrow} A$, the simulation-like result follows the pattern below, similar to a higher-order bisimilarity clause, such as the one for Plain CHOCS [26].

Let $P \mathcal{R} \bullet Q$. If $P \stackrel{\lambda}{\rightarrow} A$, then for all $\lambda \mathcal{R}^{\bullet} \lambda^{\prime}$, there exists $B$ such that $Q \stackrel{\lambda^{\prime}}{\longrightarrow} B$ and $A \mathcal{R}^{\bullet} B$.

Early bisimulations are those where all the information about a step on one side is known before providing a matching step. In the higher-order setting with concretions and abstractions, it means that when an output occurs, the abstraction that will consume the output is specified before the matching step is given. In fact, the matching step may very well be different for a given output when the abstraction considered is different. Symmetrically, in the case of an input, the matching step is chosen depending on the input and the actual concretion that is provided. In both cases, this amounts to putting the abstraction in the label in the case of an output, and the concretion in the label in case of an input. One is thus lead to prove the following simulation property.

Conjecture 1. If $P \mathcal{R} \bullet Q$, then:

- for all $P \stackrel{\tau}{\rightarrow} P^{\prime}$, there exists $Q^{\prime}$ such that $Q \stackrel{\tau}{\rightarrow} Q^{\prime}$ and $P^{\prime} \mathcal{R}^{\bullet} Q^{\prime}$;

- for all $P \stackrel{a}{\rightarrow} F$, for all $C \mathcal{R}^{\bullet} C^{\prime}$, there exists $F^{\prime}$ such that $Q \stackrel{a}{\rightarrow} F^{\prime}$ and $F \bullet C \mathcal{R}^{\bullet} F^{\prime} \bullet C^{\prime}$;

- for all $P \stackrel{\bar{a}}{\rightarrow} C$, for all $F \mathcal{R}^{\bullet} F^{\prime}$ there exists $C^{\prime}$ such that $Q \stackrel{\bar{a}}{\rightarrow} C^{\prime}$ and for all $\mathbb{E}$, we have $F \bullet \mathbb{E}\{C\} \mathcal{R}^{\bullet} F^{\prime} \bullet \mathbb{E}\left\{C^{\prime}\right\}$.

These clauses raise several issues. First, we have to find extensions of Howe's closure to abstractions and concretions which fit an early style. Even assuming such extensions, there are issues in the inductive proof of conjecture 1 with higher-order communication. The reasoning is by induction on $P \mathcal{R} \bullet Q$. Suppose we are in the parallel case, i.e., we have $P=P_{1} \mid P_{2}$ and $Q=Q_{1} \mid Q_{2}$, with $P_{1} \mathcal{R}^{\bullet} Q_{1}$ and $P_{2} \mathcal{R}^{\bullet} Q_{2}$. Suppose that we have $P \stackrel{\tau}{\rightarrow} P^{\prime}$, and the transition comes from rule HO: we have $P_{1} \stackrel{a}{\rightarrow} F, P_{2} \stackrel{\bar{a}}{\rightarrow} C$ and $P^{\prime}=F \bullet C$. We want to find $Q^{\prime}$ such that $Q \stackrel{\tau}{\rightarrow} Q^{\prime}$ and $P^{\prime} \mathcal{R} \bullet Q^{\prime}$. We also want to use the same rule HO, hence we have to find $F^{\prime}, C^{\prime}$ such that $Q \stackrel{\tau}{\rightarrow} F^{\prime} \bullet C^{\prime}$. However we cannot use the input clause of the induction hypothesis with $P_{1}, Q_{1}$ : to have a $F^{\prime}$ such that $Q_{1} \stackrel{a}{\rightarrow} F^{\prime}$, we have to find first a concretion $C^{\prime}$ such that $C \mathcal{R}^{\bullet} C^{\prime}$. We cannot use the output clause with $P_{2}, Q_{2}$ either: to have a $C^{\prime}$ such that $Q_{2} \stackrel{\bar{a}}{\rightarrow} C^{\prime}$, we have to find first an abstraction $F^{\prime}$ such that $F \mathcal{R}^{\bullet} F^{\prime}$. We cannot bypass this mutual dependency and the inductive proof of conjecture 1 fails.

Remark 3. Note that the reasoning depends more on the bisimilarity than on the calculus: the same problem occurs with early context bisimilarities for $\mathrm{HO} \pi$, Homer, and the Kell calculus. 
The intuition behind our approach. A simple way to break the mutual dependency between concretions and abstractions is to give up on the early style. An approach, used in [6], is to change the output case to a late style (hence the name, input-early, of their bisimulation): an output is matched by another output independently of the abstraction that receives it. This breaks the symmetry and allows us to proceed forward: first find the matching output $C^{\prime}$, then for this $C^{\prime}$ find the matching input using the input-early relation $\sim_{i e}$. Howe's closure is then extended to concretions $C \sim_{i e}^{\bullet} C^{\prime}$ and a simulation-like property similar to Conjecture 1 is shown, except that the output clause is changed into:

- for all $P \stackrel{\bar{a}}{\rightarrow} C$, there exists $C^{\prime}$ such that $Q \stackrel{\bar{a}}{\rightarrow} C^{\prime}$ and $C \sim_{i e}^{\bullet} C^{\prime}$.

However, in the weak case, this input-early approach does not result in a sound and complete characterization of weak barbed congruence. Definition of weak input-early bisimilarity has to be written in the delay style: internal actions are not allowed after a visible action. The delay style is necessary to keep the concretion clause independent from abstractions. It is not satisfactory since delay bisimilarities are generally not complete with respect to weak barbed congruence.

We thus propose a different approach, detailed in Section 4, that works with weak bisimulations defined in the early non-delay style. In our solution, the output clause is not late, just a little less early. More precisely, instead of requiring the abstraction before providing a matching output, we only require the process that will do the reception (that will reduce to the abstraction). This may seem a very small change, yet it is sufficient to break the symmetry. We return to the communication problem where $P_{1} \mid P_{2}$ is in relation with $Q_{1} \mid Q_{2}$. The concretion $C^{\prime}$ from $Q_{2}$ matching the $P_{2} \stackrel{\bar{a}}{\rightarrow} C$ step depends only on $P_{1}$, which is known, and not on some unknown abstraction. We can then obtain the abstraction $F^{\prime}$ from $Q_{2}$ that matches the $P_{1} \stackrel{a}{\rightarrow} F$ step. This abstraction depends fully on $C^{\prime}$, in the usual early style. Technically, we do not use concretions and abstractions anymore. In the LTS, when a communication between $P$ and $Q$ occurs, this becomes a transition from $P$ using $Q$ as a label (rule $\mathrm{HO}_{\tau}$ in Fig. 2). Higher in the derivation, the actual output from $P$ is discovered, and we switch to dealing with the input knowing exactly the output (rule $\mathrm{OuT}_{o}$ in Fig. 3). The proof of the bisimulation property for the candidate relation relies on this serialization of the LTS, which illustrates the break in the symmetry. On the other hand, the gap between a completely early relation and this one is small enough to let us prove that they actually coincide.

\section{Complementary Semantics for $\mathrm{HO} \pi \mathrm{P}$}

We define a new semantics for $\mathrm{HO} \pi \mathrm{P}$ that coincides with the contextual one, yet allows the use of Howe's method to prove the soundness of early bisimilarities. 


$$
\begin{aligned}
& a(X) P \stackrel{a, R}{\longmapsto} P\{R / X\} \quad \mathrm{IN}_{i} \quad \frac{P \stackrel{\mu}{\longmapsto} P^{\prime}}{P\left|Q \stackrel{\mu}{\longmapsto} P^{\prime}\right| Q} \operatorname{PAR}_{i \tau} \quad \frac{P \stackrel{\mu}{\longmapsto} P^{\prime}}{a[P] \stackrel{\mu}{\longmapsto} a\left[P^{\prime}\right]} \operatorname{Loc}_{i \tau} \\
& \frac{P \stackrel{\mu}{\longmapsto} P^{\prime} \quad a \neq n(\mu)}{\nu a . P \stackrel{\mu}{\longmapsto} \nu a . P^{\prime}} \operatorname{ReSTR}_{i \tau} \quad \frac{P \stackrel{\bar{a}, Q, \square}{\longmapsto} P_{b}^{\prime}}{P \mid Q \stackrel{\tau}{\longmapsto} P^{\prime}} \mathrm{HO}_{\tau}
\end{aligned}
$$

Fig. 2. Complementary LTS for $\mathrm{HO} \pi \mathrm{P}$ : Internal and Message Input Actions

\subsection{Complementary LTS}

We define a LTS $P \stackrel{\lambda}{\longmapsto} P^{\prime}$ where processes always evolve to other processes. We have three kinds of transitions: internal actions $P \stackrel{\tau}{\longmapsto} P^{\prime}$, message input $P \stackrel{a, R}{\longmapsto}$ $P^{\prime}$, and message output $P \stackrel{\bar{a}, Q, \mathbb{E}}{\longmapsto} \widetilde{b} P^{\prime}$. We call this new LTS complementary since labels $\lambda$ contain contexts complementing and interacting with $P$ for observational purposes. They are used to establish bisimilarity.

Rules for internal actions $P \stackrel{\tau}{\longmapsto} P^{\prime}$ are similar to the ones for the contextual LTS $P \stackrel{\tau}{\rightarrow} P^{\prime}$, except for higher-order communication since message output is different; we detail rule $\mathrm{HO}_{\tau}$ later. Message input $P \stackrel{a, R}{\longmapsto} P^{\prime}$ means that process $P$ may receive the process $R$ as a message on $a$ and becomes $P^{\prime}$. In the contextual style, it means that $P \stackrel{a}{\rightarrow} F$ and $P^{\prime}=F \circ R$ for some $F$. Complementary message input is simply contextual input written in the early style. We let $\stackrel{\mu}{\longmapsto}$ range over $\stackrel{\tau}{\longmapsto}$ and $\stackrel{a, R}{\longmapsto}$. For higher-order input, we define $n(a, R)=a$. Rules can be found in Figure 2 except for the symmetric counterparts of rules $\mathrm{PAR}_{i \tau}$ and $\mathrm{HO}_{\tau}$.

We now detail output actions $P \stackrel{\bar{a}, Q, \mathbb{E}}{\longmapsto}{ }_{\widetilde{b}} P^{\prime}$. Rules can be found in Fig. 3, except for the symmetric of rule $\mathrm{PAR}_{o}$. Context bisimilarity (Definition 2) compares message outputs by making them react with an abstraction $F$ and a context $\mathbb{E}$. In complementary semantics, we consider a receiving process $Q$ instead of $F$, i.e., a process able to receive the message emitted by $P$ on $a$. Transition $P \stackrel{\bar{a}, Q, \mathbb{E}}{\longmapsto}{ }_{b} P^{\prime}$ means that $P$ is put under context $\mathbb{E}$ and emits a message on $a$, which is received by $Q$ : we have $\mathbb{E}\{P\} \mid Q \stackrel{\tau}{\longmapsto} P^{\prime}$ by communication on $a$. In the contextual style, it means that there exist $F, C$ such that $P \stackrel{\bar{a}}{\rightarrow} C, Q \stackrel{a}{\rightarrow} F$, and $P^{\prime}=F \bullet \mathbb{E}\{C\}$. It is not however a simple rewrite of contextual transitions in an early style as the abstraction $F$ is not fixed by the rule. Consider rule $\mathrm{OUT}_{o}$ for message output. Unlike contextual rule ConcR, it needs a premise $Q \stackrel{a, R}{\longmapsto} Q^{\prime}$, to check that $Q$ is able to receive on $a$ the emitted process $R$. The resulting process $Q^{\prime}$ is then run in parallel with the continuation $S$ under context $\mathbb{E}$. By hypothesis of rule $\mathrm{OuT}_{o}$, the context does not capture any free name of $R$. We explain below why we choose to first deal with capture-free contexts. For such contexts, we introduce the transition $\bar{a}\langle R\rangle S \stackrel{\bar{a}, Q, \mathbb{E}}{\longrightarrow}{ }_{b} Q^{\prime} \mid \mathbb{E}\{S\}$. In the contextual semantics, we have $\bar{a}\langle R\rangle S \stackrel{\bar{a}}{\rightarrow}\langle R\rangle S$, and context bisimilarity tests $F \bullet \mathbb{E}\{\langle R\rangle S\}$ in the output clause, which is equal to $F \circ R \mid \mathbb{E}\{S\}$ for capture-free contexts. Since $Q^{\prime}$ may 


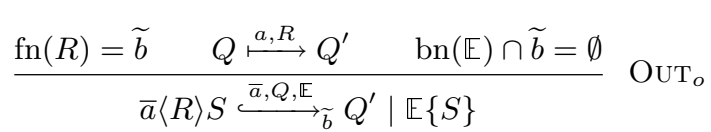

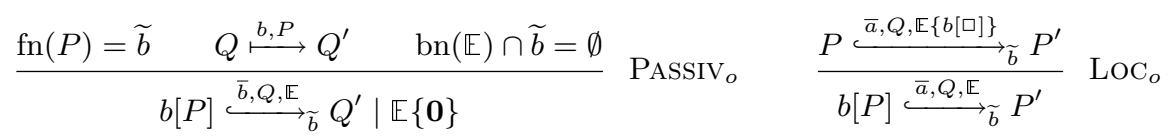

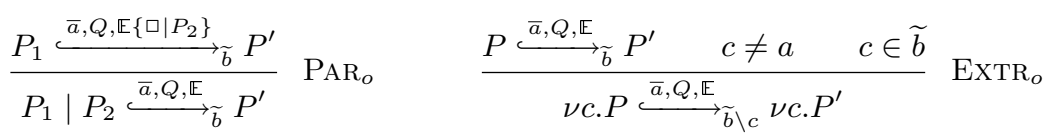

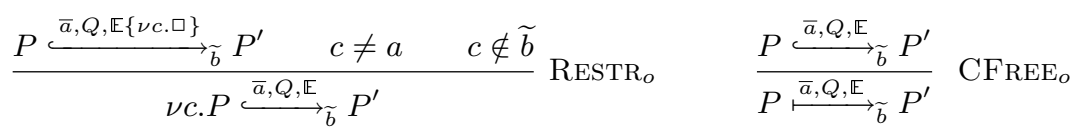

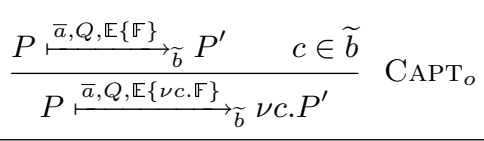

Fig. 3. Complementary LTS for $\mathrm{HO} \pi \mathrm{P}$ : Message Output Actions

be rewritten as $F \circ R$ for some $F$, the complementary transition mimics exactly the context bisimilarity output clause.

Note that the message no longer appears in the label of output transitions. We thus need additional information to deal with scope extrusion in the rules for name restriction. To this end, rule $\mathrm{OUT}_{o}$ stores the free names $\widetilde{b}$ of $R$ in the label. Scope extrusion may happen in the process under consideration (e.g., $P=\nu c . \bar{a}\langle R\rangle S$ with $c \in \mathrm{fn}(R))$ or because of the bisimulation context $\mathbb{E}$ (e.g., $P=\bar{a}\langle R\rangle S$ and $\mathbb{E}=d[\nu c .(\square \mid \bar{c}\langle\mathbf{0}\rangle \mathbf{0})]$ with $c \in \mathrm{fn}(R))$. Notice that premise $\mathrm{fn}(\mathbb{E}) \cap \widetilde{b}=\emptyset$ in rule $\mathrm{OUT}_{o}$ forbids the latter kind of capture. We thus first define auxiliary transitions $P \stackrel{\bar{a}, Q, \mathbb{E}}{\longrightarrow} P_{\tilde{b}}^{\prime}$ where $\mathbb{E}$ is a capture-free context, and we then give rules for all contexts $P \stackrel{\bar{a}, Q, \mathbb{E}}{\longmapsto}{ }_{b} P^{\prime}$.

We now explain the restriction rules on an example; let $P=\bar{a}\langle R\rangle S$ and $c \in \mathrm{fn}(R)$. Process $\nu c . P$ may emit $R$ on $a$, but the scope of $c$ has to be expanded to encompass the recipient of $R$. First premise of rule $\operatorname{ExTR}_{o}$ checks that $P$ may output a message; here we have $\bar{a}\langle R\rangle S \stackrel{\bar{a}, Q, \mathbb{E}}{\longrightarrow} \widetilde{b} \mathbb{E}\{S\} \mid Q^{\prime}$ with $\widetilde{b}=\operatorname{fn}(R)$. In conclusion, we have $\nu c . \bar{a}\langle R\rangle S \stackrel{\bar{a}, Q, \mathbb{E}}{\longrightarrow} \widetilde{b} \backslash c$ c. $\left(\mathbb{E}\{S\} \mid Q^{\prime}\right)$. Scope of $c$ includes $Q^{\prime}$ as expected. For a concretion $C=\nu \widetilde{a} .\left\langle P_{1}\right\rangle P_{2}$, the names $\widetilde{b_{C}}$ that may be extruded are the free names of $P_{1}$ which are not already bound in $\widetilde{a}$, i.e., $\widetilde{b_{C}}=\mathrm{fn}\left(P_{1}\right) \backslash \widetilde{a}$.

Suppose now that $P=\bar{a}\langle R\rangle S$ with $c \notin \mathrm{fn}(R)$. Process $\nu c . P$ may emit a message, but the scope of $c$ has to encompass only the continuation $S$ : we want to obtain $\nu c . P \stackrel{\bar{a}, Q, \mathbb{E}}{\longrightarrow} \widetilde{b} \mathbb{E}\{\nu c . S\} \mid Q^{\prime}$. To this end, we consider $P \stackrel{\bar{a}, Q, \mathbb{E}\{\nu c . \square\}}{\longrightarrow} \widetilde{b}$ 
$P^{\prime}$ as a premise of rule $\operatorname{RESTR}_{o}$. In process $P^{\prime}$, the continuation is put under $\mathbb{E}\{\nu c . \square\}$, hence we obtain $\bar{a}\langle R\rangle S \stackrel{\bar{a}, Q, \mathbb{E}\{\nu c . \square\}}{\longrightarrow} \widetilde{E}\{\nu c . S\} \mid Q^{\prime}=P^{\prime}$, as expected and reflected in the conclusion of the rule.

Rule for passivation PAssiv ${ }_{o}$ is similar to rule $\mathrm{OUT}_{o}$, while rules $\mathrm{LOC}_{o}$ and $\mathrm{PAR}_{o}$ follow the same pattern as rule RESTR . Rule CFREE $_{o}$ simply means that a transition with a capture-free context is a message output transition. We now explain how to deal with context capture with rule $\mathrm{CAPT}_{o}$. Suppose $P=\bar{a}\langle R\rangle S$ and $\mathbb{E}^{\prime}=d[\nu c .(\square \mid \bar{c}\langle\mathbf{0}\rangle \mathbf{0})]$ with $c \in \operatorname{fn}(R)$; we want to obtain $P \stackrel{\bar{a}, Q, \mathbb{E}^{\prime}}{\stackrel{b}{b}} \nu c .\left(d[S \mid \bar{c}\langle\mathbf{0}\rangle \mathbf{0}] \mid Q^{\prime}\right)$ (with the scope of $c$ extended out of $d$ ). We first consider the transition $P \stackrel{\bar{a}, Q, \mathbb{E}\{\mathbb{F}\}}{\lessgtr} \widetilde{b} P^{\prime}$ without capture on $c$; in our case we have $P \stackrel{\bar{a}, Q, d[\square \mid \bar{c}\langle\mathbf{0}\rangle \mathbf{0}]}{{ }_{\tilde{b}}} d[S \mid \bar{c}\langle\mathbf{0}\rangle \mathbf{0}] \mid Q^{\prime}=P^{\prime}$ with $\mathbb{E}=d[\square]$ and $\mathbb{F}=\square \mid \bar{c}\langle\mathbf{0}\rangle \mathbf{0}$.

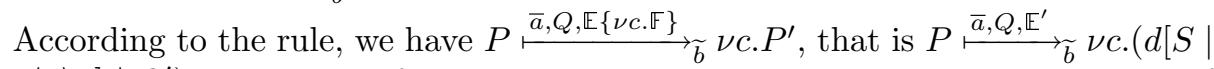
$\left.\bar{c}\langle\mathbf{0}\rangle \mathbf{0}] \mid Q^{\prime}\right)$. The scope of $c$ is extended outside $\mathbb{E}$ and includes the recipient of the message, as required.

Premise $P \stackrel{\bar{a}, Q, \square}{\stackrel{b}{b}} P^{\prime}$ of rule $\mathrm{HO}_{\tau}$ (Figure 2) means that process $P$ sends a message on $a$ to $Q$ without any context around $P$, and the result is $P^{\prime}$. Consequently we have $P \mid Q \stackrel{\tau}{\longmapsto} P^{\prime}$ by communication on $a$, which is the expected conclusion. Names $\widetilde{b}$ may no longer be potentially extruded, so we simply forget them.

\subsection{Complementary Bisimilarity}

We now define complementary bisimilarity and prove its soundness using Howe's method. Strong complementary bisimilarity for $\mathrm{HO} \pi \mathrm{P}$ is simply the classic bisimilarity associated to the complementary LTS with an additional condition on free names.

Definition 6. Strong complementary bisimilarity $\sim_{m}$ is the largest symmetric relation $\mathcal{R}$ such that $P \mathcal{R} Q$ implies $f n(P)=f n(Q)$ and for all $P \stackrel{\lambda}{\mapsto} P^{\prime}$, there exists $Q \stackrel{\lambda}{\mapsto} Q^{\prime}$ such that $P^{\prime} \mathcal{R} Q^{\prime}$.

We define $\mathbb{E} \sim_{m}^{\bullet} \mathbb{E}^{\prime}$ as the smallest congruence that extends $\sim_{m}^{\bullet}$ with $\square \sim_{m}^{\bullet} \square$. We also extend $\sim_{m}^{\bullet}$ to labels $\lambda$ in the following way: we have $\lambda \sim_{m}^{\bullet} \lambda^{\prime}$ iff $\lambda=\lambda^{\prime}=$ $\tau$, or $\lambda=(a, R), \lambda^{\prime}=\left(a, R^{\prime}\right)$ with $R \sim_{m}^{\bullet} R^{\prime}$, or $\lambda=(\bar{a}, R, \mathbb{E}, \widetilde{b}), \lambda^{\prime}=\left(\bar{a}, R^{\prime}, \mathbb{E}^{\prime}, \widetilde{b}\right)$ with $R \sim_{m}^{\bullet} R^{\prime}$ and $\mathbb{E} \sim_{m}^{\bullet} \mathbb{E}^{\prime}$. We prove the following simulation-like property for $\sim_{m}^{\bullet}:$

Lemma 2. Let $P, Q$ be closed processes. If $P \sim_{m}^{\bullet} Q$ and $P \stackrel{\lambda}{\mapsto} Q$, then for all $\lambda \sim_{m}^{\bullet} \lambda^{\prime}$, there exists $Q^{\prime}$ such that $Q \stackrel{\lambda^{\prime}}{\longmapsto} Q^{\prime}$ and $P^{\prime} \sim_{m}^{\bullet} Q^{\prime}$.

The higher-order communication problem of Section 3 is avoided. We recall that in this case, we have $P_{1}\left|P_{2} \sim_{m}^{\bullet} Q_{1}\right| Q_{2}$ with $P_{1} \sim_{m}^{\cdot} Q_{1}, P_{2} \sim_{m}^{\bullet} Q_{2}$ and $P_{1} \stackrel{\bar{a}, P_{2}, \square}{\longrightarrow} P^{\prime}$. We can apply directly the message output clause of the induction 
hypothesis: there exists $Q^{\prime}$ such that $Q_{1} \stackrel{\bar{a}, Q_{2}, \square}{\longrightarrow}{ }_{\tilde{b}} Q^{\prime}$ and $P^{\prime} \sim_{m}^{\bullet} Q^{\prime}$. We conclude that $Q_{1} \mid Q_{2} \stackrel{\tau}{\mapsto} Q^{\prime}$ (by rule $\mathrm{HO}_{\tau}$ ) with $P^{\prime} \sim_{m}^{\bullet} Q^{\prime}$ as wished.

Theorem 2. Relation $\sim_{m}$ is a congruence.

We now turn to the relationship between context and complementary bisimilarities. We show that they actually coincide.

Theorem 3. We have $\sim=\sim_{m}$.

In the message input case, complementary bisimilarity tests with a process while context bisimilarity tests with a concretion. Both testings are equivalent because of the congruence of $\sim_{m}$ (Theorem 2). See [13] for more details. The output clause of complementary bisimilarity requires that transition $P \stackrel{\bar{a}, T, \mathbb{E}}{\stackrel{b}{b}} P^{\prime}$ has to be matched by a transition $Q \stackrel{\bar{a}, T, \mathbb{E}}{\rightleftharpoons} \widetilde{b} Q^{\prime}$ with the same set of names $\widetilde{b}$ which may be extruded. At first glance, we do not have this requirement for the early strong context bisimilarity, hence we first have to prove that it is the case before proving Theorem 3 .

Correspondence also holds in the weak case. We write $\stackrel{\tau}{\Leftrightarrow}$ the reflexive and transitive closure of $\stackrel{\tau}{\mapsto}$. We define $\stackrel{a, R}{\Longleftrightarrow}$ as $\stackrel{\tau}{\mapsto} \stackrel{a, R}{\longmapsto} \stackrel{\tau}{\mapsto}$. In the weak case, two processes $P$ and $Q$ may evolve independently before interacting with each other. Since a transition $P \stackrel{\bar{a}, Q, \mathbb{E}}{\stackrel{b}{b}} P^{\prime}$ includes a communication between $P$ and $Q$, we have to authorize $Q$ to perform $\tau$-actions before interacting with $P$ in the weak

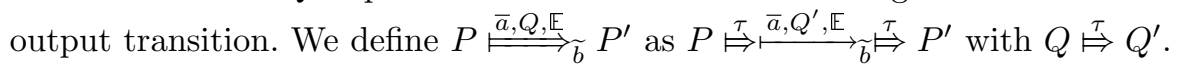

Definition 7. Weak complementary bisimilarity $\approx_{m}$ is the largest symmetric relation $\mathcal{R}$ such that $P \mathcal{R} Q$ implies $f n(P)=f n(Q)$ and for all $P \stackrel{\lambda}{\longmapsto} P^{\prime}$, there exists $Q \stackrel{\lambda}{\Rightarrow} Q^{\prime}$ such that $P^{\prime} \mathcal{R} Q^{\prime}$.

Using the same proofs techniques as in the strong case, we have the following results.

Theorem 4. Relation $\approx_{m}$ is a congruence.

Theorem 5. We have $\approx_{m}=\approx$.

Bisimilarity $\approx_{m}$ coincides with $\approx_{b}$ on image-finite processes. The limitation on image-finite processes is usual and can be found in $\pi$-calculus [23] for instance. A closed process $P$ is image finite iff for every label $\lambda$, the set $\left\{P^{\prime}, P \stackrel{\lambda}{\Leftrightarrow} P^{\prime}\right\}$ is finite. Using the same proof technique as in [23], we have the following completeness result.

Theorem 6. Let $P, Q$ be image-finite processes. $P \approx_{b} Q$ if and only if $P \approx_{m} Q$. 


\section{Related Work}

Howe's method This method has been used originally to prove congruence in a lazy functional programming language [10]. Baldamus and Frauenstein [2] are the first to adapt Howe's method to process calculi. They prove congruence of a late delay context bisimilarity, and of late and early delay higher-order bisimilarities in variants of Plain CHOCS [26]. Hildebrandt and Godskesen use Howe's method for their calculus Homer to prove congruence for late delay [9] and input-early delay [6] context bisimilarities. In [15], we have used Howe's method to prove congruence of a weak higher-order bisimilarity for a calculus featuring passivation but without restriction.

Behavioral equivalences in higher-order calculi Very few higher-order calculi feature a coinductive characterization of weak barbed congruence. It is the case in $\mathrm{HO} \pi$ and in variants of Mobile Ambients. Sangiorgi introduces context bisimilarities as characterizations of barbed congruence for its calculus $\mathrm{HO} \pi$ [21]. Mobile Ambients [4] is a calculus with hierarchical localities and subjective linear process mobility. Contextual characterizations of weak barbed congruence have been defined for Mobile Ambients [16] and its variant NBA [3].

Difficulties arise in more expressive process calculi. The Seal calculus [5] is a calculus with objective, non linear process mobility, which requires synchronization between three processes (a process sending a name $a$, a receiving process, and a locality named $a$ ). Castagna et al. define a sound weak delay context bisimilarity in [5] called Hoe bisimilarity for the Seal calculus. The authors point out that their notion of context bisimilarity, Hoe bisimilarity, is not complete, not only because of the delay style, but also because of the labels introduced for partial synchronization which are most likely not all observable.

The Kell calculus [24] and Homer [9] are two higher-order calculi featuring passivation. They differ in how they handle communication; in particular, the Kell calculus allows join patterns while Homer does not. Sound and complete context bisimilarities have been defined for both calculi in the strong case. As stated before, a weak delay input-early bisimilarity has been proved sound in Homer using Howe's method. In [15] we have studied various calculi with passivation in order to find normal bisimilarities for those calculi, i.e., relations with finite testing at each step. In particular we have studied $\mathrm{HO} \pi \mathrm{P}$ and showed that its behavioral theory raises similar difficulties as the Homer and Kell ones.

One can also establish soundness by writing transitions in a special rule format that ensures that the corresponding bisimilarity is a congruence. For higher-order calculi, Mousavi et al. [18] propose Promoted (resp. Higher-Order) PANTH format which guarantees that the corresponding strong (resp. higherorder) bisimilarity is a congruence. Another method is to generate the LTS from the reduction such that the corresponding bisimilarity is a congruence [19]. To this date, both methods have been applied in the strong case only. One can also prove congruence using environmental bisimulations [22]. We have not been able to apply this method to a calculus with passivation yet. 


\section{Conclusion and Future Work}

Contextual LTS (based on abstractions and/or concretions) are not well suited to prove congruence using Howe's method. The method relies on a simulationlike property, which is hard to establish with early context bisimilarities that are the usual candidate relations for characterization of barbed congruence. The main issue is the mutual dependency between message input and output clauses of the context bisimilarity. In our complementary semantics, the message output clause depends on a process which may receive the message (i.e., a process which evolves towards an abstraction), instead of an abstraction which directly receives the message. This proof technique allows Howe's method to work with early weak (non delay) bisimilarity.

We have defined complementary semantics for $\mathrm{HO} \pi \mathrm{P}$, an extension of $\mathrm{HO} \pi$ with passivation. In Section 4, we have defined a weak complementary bisimilarity and proved its soundness using Howe's method. We have also proved that it coincides with weak barbed congruence on image-finite processes, yielding the first characterization result in a calculus featuring passivation and restriction. This approach may be applied to other calculi: in [13] we present a sound weak complementary bisimilarity for $\mathrm{HO} \pi$ and for the Seal calculus [5].

An immediate future work would be to define a complementary semantics for process calculi with no characterization result for weak barbed congruence, such as Seal, Homer [6], and the Kell calculus [24], and to prove that complementary bisimilarity in these calculi yields the required characterization. It should be easy for Homer since the $\mathrm{HO} \pi \mathrm{P}$ semantics is close to the Homer one. For Seal, it remains to show that the complementary semantics in [13] is indeed complete. The Kell calculus may prove to be more challenging because of join patterns: to complement an emitting process $P$, we need a receiving process $Q$, but also other emitting processes $\widetilde{R}$ to match the receiving pattern of $Q$. Another future work is to define a LTS rule format which guarantees that Howe's method works with the corresponding bisimilarity, possibly extending the Promoted or Higher-Order PANTH format for higher-order calculi proposed by Mousavi et al. [18].

\section{References}

1. M. Baldamus. Semantics and Logic of Higher-Order Processes: Characterizing Late Context Bisimulation. PhD thesis, Berlin University of Technology, 1998.

2. M. Baldamus and T. Frauenstein. Congruence proofs for weak bisimulation equivalences on higher-order process calculi. Technical report, Berlin University of Technology, 1995.

3. M. Bugliesi, S. Crafa, M. Merro, and V. Sassone. Communication and mobility control in boxed ambients. Information and Computation, 202, 2005.

4. L. Cardelli and A. D. Gordon. Mobile ambients. In FoSSaCS '98, volume 1378 of LNCS, pages 140-155. Springer, 1998.

5. G. Castagna, J. Vitek, and F. Zappa Nardelli. The Seal Calculus. Information and Computation, 201(1):1-54, 2005. 
6. J. C. Godskesen and T. Hildebrandt. Extending howe's method to early bisimulations for typed mobile embedded resources with local names. In FSTTCS '05, volume 3821 of $L N C S$, pages 140-151. Springer, 2005.

7. A. D. Gordon. Bisimilarity as a theory of functional programming. Mini-course. Notes Series NS-95-3, BRICS, University of Cambridge Computer Laboratory, July 1995. iv $+59 \mathrm{pp}$

8. M. Hennessy, J. Rathke, and N. Yoshida. Safedpi: a language for controlling mobile code. Acta Inf., 42(4-5), 2005.

9. T. Hildebrandt, J. C. Godskesen, and M. Bundgaard. Bisimulation congruences for Homer - a calculus of higher order mobile embedded resources. Technical Report ITU-TR-2004-52, IT University of Copenhagen, 2004.

10. D. J. Howe. Proving congruence of bisimulation in functional programming languages. Information and Computation, 124(2):103-112, 1996.

11. A. Jeffrey and J. Rathke. A theory of bisimulation for a fragment of concurrent ML with local names. Theoretical Computer Science, 323:1-48, 2004.

12. A. Jeffrey and J. Rathke. Contextual equivalence for higher-order pi-calculus revisited. Logical Methods in Computer Science, 1(1), 2005.

13. S. Lenglet, A. Schmitt, and J.B.Stefani. Howe's method for early bisimilarities. Technical Report RR 6773, INRIA, 2008.

14. S. Lenglet, A. Schmitt, and J.B.Stefani. Characterizing contextual equivalence in calculi with passivation. draft available at http://sardes.inrialpes.fr/ aschmitt/papers/hop_howe_long.pdf, 2009.

15. S. Lenglet, A. Schmitt, and J.-B. Stefani. Normal bisimulations in process calculi with passivation. In FoSSaCS '09, volume 5504 of $L N C S$, pages 257-271. Springer, 2009.

16. M. Merro and F. Zappa Nardelli. Behavioral theory for mobile ambients. Journal of the ACM, 52(6):961-1023, 2005.

17. R. Milner and D. Sangiorgi. Barbed bisimulation. In ICALP '92, volume 623 of LNCS. Springer, 1992.

18. M. Mousavi, M. J. Gabbay, and M. A. Reniers. Sos for higher order processes (extended abstract). In CONCUR'05, volume 3653 of $L N C S$, pages 308-322. Springer, 2005.

19. J. Rathke and P. Sobocinski. Deconstructing behavioural theories of mobility. In IFIP TCS, volume 273 of IFIP, pages 507-520. Springer, 2008.

20. D. Sangiorgi. Expressing Mobility in Process Algebras: First-Order and HigherOrder Paradigms. PhD thesis, Department of Computer Science, University of Edinburgh, 1992.

21. D. Sangiorgi. Bisimulation for higher-order process calculi. Information and Computation, 131(2):141-178, 1996.

22. D. Sangiorgi, N. Kobayashi, and E. Sumii. Environmental bisimulations for higherorder languages. In LICS '07, pages 293-302. IEEE Computer Society, 2007.

23. D. Sangiorgi and D. Walker. The Pi-Calculus: A Theory of Mobile Processes. Cambridge University Press, 2001.

24. A. Schmitt and J.-B. Stefani. The Kell Calculus: A Family of Higher-Order Distributed Process Calculi. In Global Computing 2004 workshop, volume 3267 of LNCS, 2004.

25. P. Sewell, J. Leifer, K. Wansbrough, F. Zappa Nardelli, M. Allen-Willians, P. Habouzit, and V. Vafeiadis. Acute: High-level programming language design for distributed computation. Journal of Functional Programming, 17(4-5), 2007.

26. B. Thomsen. Plain chocs: A second generation calculus for higher order processes. Acta Informatica, 30(1):1-59, 1993. 